\title{
Enhanced fluorescence from X-ray line coincidence pumping
}

\author{
Joseph Nilsen $^{1}$, D. Burridge 2 , L. M. R. Hobbs ${ }^{2}$, D. Hoarty ${ }^{2}$, P. Beiersdorfer ${ }^{1}$, \\ G. V. Brown ${ }^{1}$, N. Hell ${ }^{1}$, D. Panchenko ${ }^{1}$, M. F. Gu ${ }^{3}$, A. M. Saunders ${ }^{1}$, H. A. \\ Scott $^{1}$, P. Hatfield ${ }^{4}$, M. P. Hill ${ }^{2}$, L. Wilson ${ }^{2}$, R. Charles ${ }^{2}$, C. R. D. Brown ${ }^{2}$, and \\ S. Rose ${ }^{4}$ \\ ${ }^{1}$ Lawrence Livermore National Laboratory, Livermore, CA 94551 \\ ${ }^{2} \mathrm{AWE}$ Plc, UK \\ ${ }^{3}$ University of California, Berkeley, CA \\ ${ }^{4}$ Imperial College, UK
}

\begin{abstract}
Many resonant photo-pumped X-ray laser schemes that use a strong pump line such as Ly- $\alpha$ or He- $\alpha$ to populate the upper laser state of a separate lasing material have been proposed over the last four decades but none have been demonstrated. As a first step to creating a photo-pumped X-ray laser we have decided to reinvestigate some of these schemes at the Orion laser facility with the goal to show enhanced fluorescence. In particular we look at using the $\mathrm{Ly}-\alpha$ or $\mathrm{He}-\alpha \mathrm{K}$ lines to pump the $1 \mathrm{~s}-$ $3 \mathrm{p}$ and $4 \mathrm{p}$ transitions in $\mathrm{H}$-like $\mathrm{Cl}$ and see fluorescence on the $4 \mathrm{f}-3 \mathrm{~d}$ line at $65 \AA$ and the $3 \mathrm{~d}-2 \mathrm{p}$ line at $23 \AA$. Preliminary experiments are presented that show a modest enhancement. As an alternative we also look at enhancing the $2 p-2$ s line in Ne-like Ge at $65 \AA$ using the Ly- $\alpha \mathrm{Mg}$ line to photo-pump the $2 \mathrm{~s}-3 \mathrm{p}$ line of Ne-like Ge. Calculations are presented that suggest modest enhancements of 2.5.
\end{abstract}

\section{Introduction}

One early approach for creating X-ray lasers was the idea of a resonantly photo-pumped laser where a strong emission line in one material could be used to photo-excite a transition in another material and create lasing. The Napumped $\mathrm{Ne} \mathrm{X}$-ray laser scheme proposed 40 years ago by Vinogradov and colleagues $[1,2]$ is a classic example that used the strong $\mathrm{Na} \mathrm{He}-\alpha$ line at 1127 $\mathrm{eV}$ to resonantly photo-pump the $\mathrm{Ne} \mathrm{He}-\gamma$ line and lase on the $4 \mathrm{f}-3 \mathrm{~d}$ transition at $231 \AA$ in He-like Ne. This scheme was studied extensively and weak gain [3] was inferred in several experiments. There are a series of similar resonances [2] that use Ly- $\alpha$ or He- $\alpha$ lines to photo-pump Ly- $\gamma$ or He- $\gamma$ lines and lase on the $4 \mathrm{f}-3 \mathrm{~d}$ lines of $\mathrm{H}$ or He-like ions. In this paper we discuss the next higher $\mathrm{Z}$ pair of possible resonances that use the strong Ly- $\alpha$ line at $3699 \mathrm{eV}$ from $\mathrm{H}-$ like $\mathrm{K}$ 
to resonantly photo-pump the $1 \mathrm{~s}-4 \mathrm{p}$ transition in $\mathrm{Cl}$ and enhance the emission of the $4 \mathrm{f}-3 \mathrm{~d}$ line at $65 \AA$ in $\mathrm{H}$-like $\mathrm{Cl}$.

One can also pump the Ly- $\beta$ or He- $\beta$ lines and lase or enhance the fluorescence of the $3 \mathrm{~d}-2 \mathrm{p}$ lines. The $\mathrm{K}-\mathrm{Cl}$ combination is interesting because it also has a resonance between the strong $\mathrm{K} \mathrm{He}-\alpha$ line to resonantly photopump the $\mathrm{Cl} \mathrm{He}-\beta$ transition and enhance the emission of the $3 d-2 p$ line at 23 $\AA$ in $\mathrm{H}$-like $\mathrm{Cl}$.

Experiments were done at the Orion laser facility in the UK to look at enhanced emission on both the 23 and $65 \AA \mathrm{Cl}$ lines.

An alternative experiment is being designed to look at the emission of the $2 p$ - 2s line in Ne-like Ge at $65 \AA$ that could be photo-pumped by the Ly- $\alpha$ line of $\mathrm{H}$-like $\mathrm{Mg}$ at $1472 \mathrm{eV}$. In this case the Ly- $\alpha$ line photo-pumps the inner shell $2 s-3 p$ ground state transition. This resonance was measured in previous experiments at the EBIT facility at LLNL, the NPO laser facility at VNIIFTRI, and the Princeton Large Torus (PLT) tokamak. Experiments are being planned at the Orion laser facility to investigate this scheme.

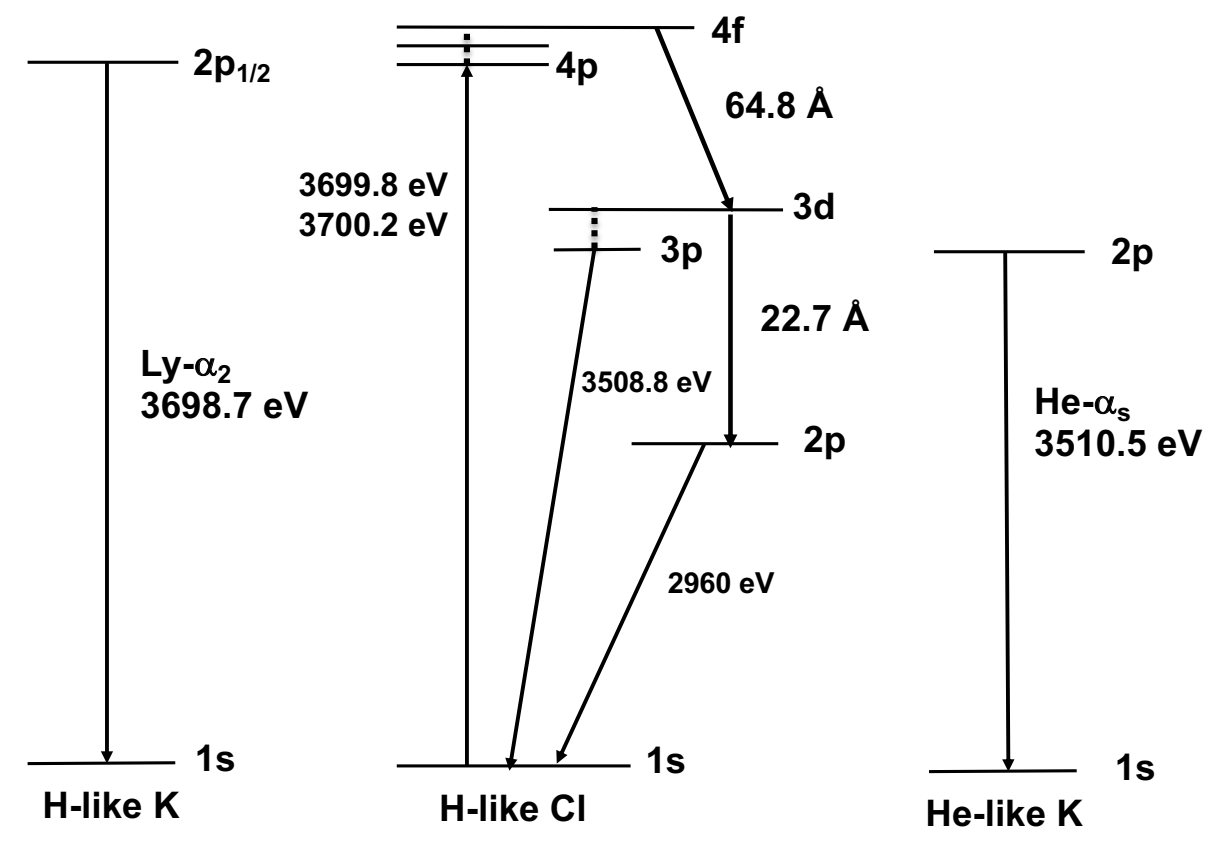

Fig. 1. Energy level diagram for the Ly- $\alpha$ and He- $\alpha$ lines of K photo-pumping the $1 \mathrm{~s}-3 \mathrm{p}$ and $4 \mathrm{p}$ lines of $\mathrm{H}$-like $\mathrm{Cl}$ to enhance the fluorescence of the $4 \mathrm{f}-3 \mathrm{~d}$ line at $64.8 \AA$ and the $3 \mathrm{~d}-2 \mathrm{p}$ line at $22.7 \AA$. 


\section{K-pumped Cl experiments}

Figure 1 shows the energy level diagram for the Potassium (K)-pumped Chlorine $(\mathrm{Cl})$ schemes. The $4 \mathrm{f}-3 \mathrm{~d}$ line at $64.8 \AA$ in $\mathrm{H}$-like $\mathrm{Cl}$ is enhanced by having the strong Ly- $\alpha$ line of K photo-pump the 1s $-4 p(L y-\gamma)$ transition of $\mathrm{H}$-like $\mathrm{Cl}$. The $\mathrm{n}=4$ states of $\mathrm{Cl}$ are all very close in energy and become statistically populated by collisional processes at the temperatures and densities expected in the plasma. The $4 \mathrm{f}$ level has the largest statistical weight $(2 \mathrm{~J}+1)$ which results in strong emission and potential gain on the $4 \mathrm{f}-3 \mathrm{~d}$ line. Potassium is rather unique because the $\mathrm{He}-\alpha$ line, which is usually the strongest line one can produce in a hot plasma, is nearly resonant with the $1 \mathrm{~s}-3 \mathrm{p}$ (Ly$\beta$ ) line of $\mathrm{Cl}$. This photo-pumps population into the $3 p$ level which then equilibrates through collisional processes to statistically populate the $n=3$ level resulting in a large population in the $3 \mathrm{~d}$ level. One then looks for strong emission on the $3 d-2 p$ line at $22.7 \AA$. This line is also part of the cascade from the $4 \mathrm{f}-3 \mathrm{~d}$ emission which also enhances the line. This scheme was originally proposed and modelled $[3,4]$ many years ago.

To look for potential enhancements, eexperiments were done at the Orion laser facility [5] at the atomic weapons establishment (AWE) in the UK. The laser systems consist of ten beams, optimized around $1 \mathrm{~ns}$ pulse duration, which each provide a nominal $500 \mathrm{~J}$ at a wavelength of $351 \mathrm{~nm}$. There are also two short pulse beams, which each provide $500 \mathrm{~J}$ in $0.5 \mathrm{ps}$ at $1054 \mathrm{~nm}$, that were not used in these experiments.

The targets consisted a $0.5 \mu \mathrm{m}$ thick microdot of $\mathrm{KCl}$ with a diameter of 300 $\mu \mathrm{m}$. This was coated on each side with $0.5 \mu \mathrm{m}$ thick layer of KF and then an



Fig. 2. Emission from the $\mathrm{NaCl}$ target and $\mathrm{KCl}$ target showing the strong $\mathrm{Ly}-\alpha$ and $\mathrm{He}-\alpha$ emission lines from $\mathrm{K}$ and $\mathrm{Cl}$. 
outer protective coating of $0.3 \mu \mathrm{m}$ thick layer of parylene-N, which is a $\mathrm{CH}$ plastic polymer. The goal of the KF layer was to have a thick layer of $\mathrm{K}$ surrounding the $\mathrm{Cl}$ plasma that would enhance the strength of the Ly- $\alpha$ and He$\alpha \mathrm{K}$ lines pumping the $\mathrm{Cl}$. As a null experiment we used targets that replaced the $\mathrm{KCl}$ with $\mathrm{NaCl}$ and the $\mathrm{KF}$ with $\mathrm{NaF}$. In the null experiment there are no $\mathrm{K}$ lines to photo-pump the $\mathrm{Cl}$ plasma.

The targets were hit from both side by $250-1150 \mathrm{~J}$ of energy in a $300 \mu \mathrm{m}$ diameter beam with a 1-1.5 ns FWHM pulse duration. Hydrodynamic calculations done with the 1D NYM code predict that the plasma would expand and blow down to densities of about 1-2 mg/cc or electron density of 3-6 x $10^{20} / \mathrm{cc}$ and reach temperatures up to several $\mathrm{keV}$. Under these conditions we expect to ionize the plasmas to $\mathrm{H}$ and He-like states. We observe strong emission, as seen in Fig. 2, on the $\mathrm{K}$ and $\mathrm{Cl} \mathrm{Ly}-\alpha$ and He- $\alpha$ lines for the nominal target and strong emission on the $\mathrm{Cl} \mathrm{Ly}-\alpha$ and $\mathrm{He}-\alpha$ lines for the null target indicating that we have reached the correct ionization conditions. This data was recorded on the Mark 2 time-integrated spectrometer with a resolution of $7 \mathrm{eV}$ using CsAP crystals.

To look at the spectral lines near 23 and $65 \AA$ we used a grating instrument spectrometer that covered the range of $100-1000 \mathrm{eV}(120-12 \AA)$ with a resolution of 1000 . Figure 3 shows the spectrum from 21-27 $\AA$ that looks at the enhancement of the $\mathrm{Cl} 3 \mathrm{~d}-2 \mathrm{p}$ line at $22.7 \AA$. When we normalize to the other $\mathrm{Cl}$ lines we believe we observe a modest enhancement of about $20 \%$ on this line due to the photo-pumping. The spectrum near the $65 \AA$ line is more

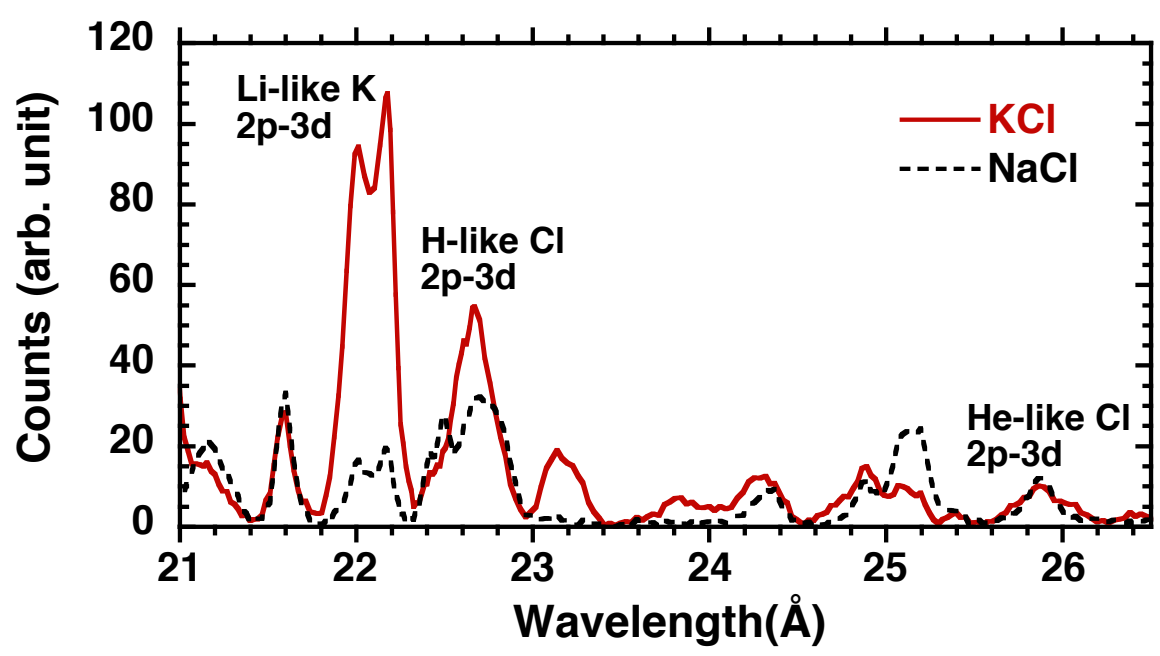

Fig. 3. Emission from the $\mathrm{NaCl}$ and $\mathrm{KCl}$ targets shows modest enhancement of the $\mathrm{H}$-like $\mathrm{Cl} 3 \mathrm{~d}-2 \mathrm{p}$ line at $22.7 \AA ̊$ in the $\mathrm{KCl}$ target. 
complicated and still being analysed as there are nearby lines in Li-like $\mathrm{Cl}$ in the same spectral region.

\section{Mg-pumped Ge experiments}

The second scheme that we are actively pursuing, shown in Fig. 4, uses the Magnesium (Mg) Ly- $\alpha$ lines to photo-pump the Neon (Ne)-like Germanium (Ge) $2 s-3 p$ line (B) and enhance the emission of the $2 p-2 s$ line in Ne-like $\mathrm{Ge}$ at $65.1 \AA$. The resonance between the $\mathrm{Mg}$ and $\mathrm{Ge}$ lines were measured in previous experiments at the EBIT facility at LLNL, the NPO laser facility at VNIIFTRI, and the Princeton Large Torus (PLT) tokamak. [6] Experiments are being planned at the Orion laser facility to investigate this scheme.

These experiments would be very similar to the KCL experiments where we use microdot targets with a $0.2 \mu \mathrm{m}$ thick Ge target surrounded by $0.3 \mu \mathrm{m}$ of $\mathrm{Mg}$ for the pumped experiment and $0.3 \mu \mathrm{m}$ of $\mathrm{Al}$ for the null experiment. Both sets of targets are overcoated with $0.3 \mu \mathrm{m}$ of Parylene- $\mathrm{N}$ on each side as a protective layer from the atmosphere. To investigate the viability of this scheme we have done a series of kinetics and radiation transport calculations with the Cretin

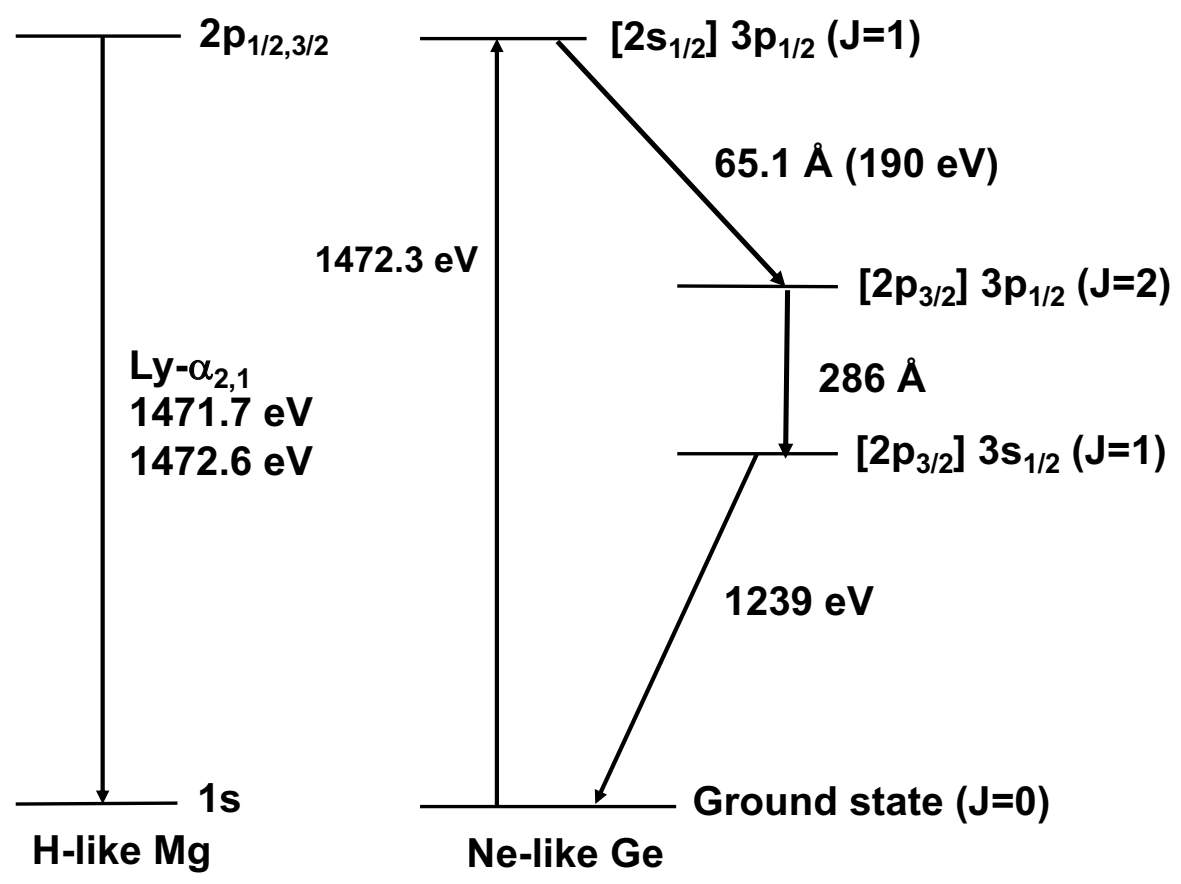

Fig. 4. Energy level diagram for the Mg Ly- $\alpha$ photo-pumping the Ne-like Ge $2 \mathrm{~s}-$ $3 p$ line to enhance the fluorescence of the $2 p-2$ s line at $65.1 \AA$ 


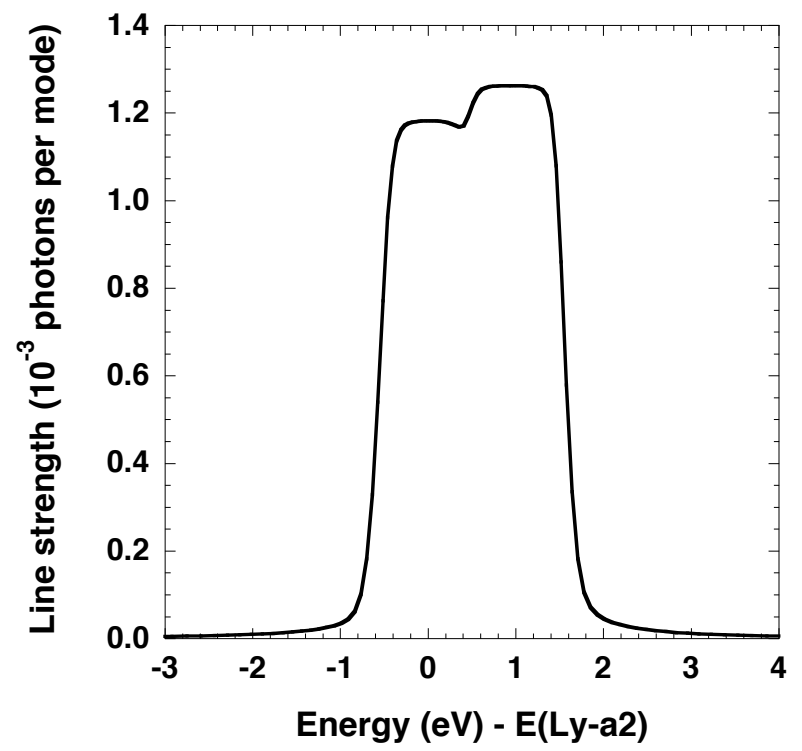

Fig. 5. Line strength for the Mg Ly- $\alpha$ line vs photon energy centered around the Ly- $\alpha 2$ line.

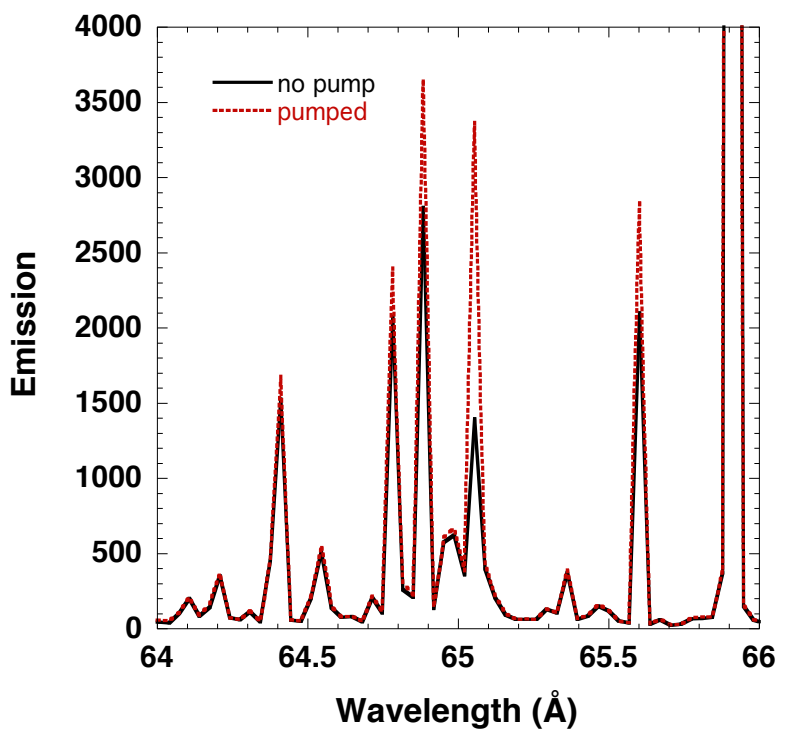

Fig. 6. Predicted emission from the Ge target comparing the case where it is pumped by the Mg Ly- $\alpha$ line to the case without any pumping.

code [7] to estimate enhancements that we might see in the upcoming 
experiments at the Orion facility.

If we assume the targets have blown down in density to an electron density of $5 \times 10^{20} / \mathrm{cc}(1.8 \mathrm{mg} / \mathrm{cc})$ at a temperature of $500 \mathrm{eV}$ we can first estimate the strength and width of the Mg Ly- $\alpha$ alpha line. Doing a 1D line transport calculation assuming a $\mathrm{Mg}$ target with a radius of $350 \mu \mathrm{m}$, Fig 5 shows the line strength for the Mg Ly- $\alpha$ in the center of the target. One sees that the line has a width of $2.1 \mathrm{eV}$ with the $\mathrm{Ge}$ line located at an energy of $0.6 \mathrm{eV}$ relative to the Ly- $\alpha 2$ line. This makes for essentially a perfect resonance between the $\mathrm{Mg}$ and Ge lines. The Mg line has a peak strength of $1.2 \times 10^{-3}$ photons per mode in the center of the target and falls off by a factor of 6 at the outer surface of the target. When pumping a line, the line strength in photons per mode is very similar to the fractional population of the upper state being pumped.

The next step is to do a kinetics calculation for the spectra of Ge near $65 \AA$ with and without the presence of the $\mathrm{Mg}$ line using the pump strength calculated above. To best estimate the spectra, we created an atomic model for Ge that uses many body perturbation theory calculations for the energy levels of $\mathrm{Ne}$ like $\mathrm{Ge} n=2$ and $n=3$ levels. These calculations have been compared with detail spectra taken at the EBIT facility [8] at LLNL.

Taking the same plasma conditions described above for Ge we use Cretin to calculate the Ge spectra. Without the Mg pump present we observe a series of lines that are dominated by the very strong F-like Ge $2 p-2$ s line at $65.9 \AA$. When we do a similar calculation including the Mg pump line the spectra are very similar except for a significant enhancement of about 2.5 on the $2 p-2 s$ Ne-like Ge line at $65.06 \AA$, as shown in Fig. 6. This is the type of enhancement we are trying to observe in the future experiments. One advantage of looking for enhanced fluorescence instead of gain is that it can take a more substantial enhancement of the line before it has enough gain to lase.

\section{Conclusions}

In this paper we look at using the $\mathrm{K} \mathrm{Ly}-\alpha$ or $\mathrm{He}-\alpha$ lines to photo-pump the $1 \mathrm{~s}-3 \mathrm{p}$ and $4 \mathrm{p}$ transitions in $\mathrm{H}$-like $\mathrm{Cl}$ and see fluorescence on the $4 \mathrm{f}-3 \mathrm{~d}$ line at $64.8 \AA$ and the $3 \mathrm{~d}-2 \mathrm{p}$ line at $22.7 \AA$. Preliminary experiments are presented that show a modest enhancement of about $20 \%$ on the $22.7 \AA$ line. As an alternative we also look at enhancing the $2 p-2$ s line in Ne-like Ge at $65.1 \AA$ using the Mg Ly- $\alpha$ line to photo-pump the $2 s-3 p$ line of Ne-like Ge. Calculations are presented that suggest modest enhancements of 2.5. Experiments are planned in the near future to test the $\mathrm{Mg}$-pumped $\mathrm{Ge}$ scheme. 
Acknowledgements. This work was performed under the auspices of the U.S. Department of Energy by Lawrence Livermore National Laboratory under Contract DE-AC52-07NA27344. Add AWE or other acknowledgments.

\section{References}

1. Vinogradov, A. V., Sobelman, I. I., Yukov, E. A., Sov. J. Quantum Electron. 5, 5963, 1975.

2. Nilsen, J., Scofield, J. H., Chandler, E. A., Appl. Opt. 31, 4950 - 4956, 1992.

3. Nilsen J., Chandler, E. A., Phys. Rev. A 44, 4591-4598, 1991.

4. Rose, S. J., J. Phys. B 31, 2129-2144, 1998.

5. Hopps, N. et al., Plasma Phys. Control Fusion 57, 064002, 2015.

6. Nilsen, J. et al., Phys. Rev A 50, 2143, 1994.

7. Scott, H. A., JQSRT 71, 689-701, 2001.

8. Beiersdorfer, P., Nuclear Instruments and Methods in Physics Research B 205, 173, 2003. 